\title{
EDITORIAL
}

\section{How children with asthma breathe: have we been overlooking a problem?}

\author{
Vikas Goyal and Peter D. Sly
}

$\mathbf{A}$ sthma is one of the most common chronic diseases globally, with an estimated 300 million sufferers [1] and is diagnosed in up to $10 \%$ of paediatric populations in many developed countries. Despite many advances in the treatment of asthma, there remain a percentage of patients, including children, who do not respond, or respond poorly to current treatment. These are the patients for whom clinicians think of alternative diagnoses.

THOMAs et al. [2] have investigated abnormal breathing patterns in adults with asthma using the Nijmegen questionnaire and have identified as many as $29 \%$ of patients as having dysfunctional breathing. Symptoms suggestive of dysfunctional breathing include: dyspnoea, deep sighing, chest pain, chest tightness, frequent yawning, hyperventilation and breathlessness during exercise [2]. The same authors also showed a positive response with breathing retraining in a randomised control trial [3]. Some others have reported some ongoing benefit of breathing retraining in patients with dysfunctional breathing in a 5-year follow-up [4]. Thus both the problem of dysfunctional breathing in adults diagnosed with asthma and the benefit such patients obtain from breathing retraining is reasonably well established.

Psychological problems in children diagnosed with asthma have been described [5-8]. Various authors have suggested that the mainstay of the diagnosis of psychogenic and functional breathing disorders is a full and meticulously taken history [7, 9]. GRÜBER et al. [9] have proposed an approach to define and classify somatoform respiratory disorders in children. In addition, Lu et al. [10] have recently published results of a metaanalysis and meta-regression designed to determine whether anxiety and depressive symptoms are more prevalent in adolescents with asthma than in their non-asthmatic counterparts. These authors concluded that the prevalence of depressive $(0.27,95 \%$ CI $0.18-0.39$ versus $0.13,0.09-0.19)$ and anxiety $(0.33,95 \%$ CI $0.19-0.52$ versus $0.21,0.12-0.33)$ symptoms was higher among 3546 adolescents with asthma than in 24884 controls [10]. In addition, the risk of developing depression and anxiety was significantly higher in adolescent asthmatics when compared with controls (depression OR 2.09, 95\% CI 1.65-2.64

Queensland Children's Medical Research institute, The University of Queensland, Brisbane, and Dept of Respiratory Medicine, Royal Children's Hospital, Brisbane, Australia.

CORRESPONDENCE: P.D. Sly, University of Queensland, Level 4, Foundation Building, Royal Children's Hospital, Brisbane, Queensland, 4029, Australia. E-mail: p.sly@uq.edu.au $(\mathrm{p}<0.001)$; anxiety OR 1.83, 95\% CI 1.63-2.07 ( $<<0.001))$, [10]. These data confirm the clinical impression that psychological problems can and do occur in children with asthma. The symptoms suggestive of dysfunctional breathing detected by the Nijmegen questionnaire are similar to those one would expect in an anxious child.

In this issue of European Respiratory Journal, DE GROOT et al. [11] used the Nijmegen questionnaire to determine the prevalence of dysfunctional breathing in children with asthma and its impact on asthma control using the paediatric asthma control questionnaire. They administered these questionnaires to 206 consecutive children attending the Princess Amalia hospital clinics (Zwolle, the Netherlands) for a scheduled asthma consultation and reported a prevalence of dysfunctional breathing of $5 \%$. They also reported that poor asthma control (asthma control questionnaire $>1.0$ ) was more common in children with higher scores on the Nijmegen questionnaire $(p<0.001)$ [11]. These findings suggest that a small proportion of children fit the adult criteria of dysfunctional breathing and that these children have worse asthma control.

However, there are a number of technical questions that need to be considered. DE GROOT et al. [11] have assumed that the Nijmegen questionnaire can be used in children to define dysfunctional breathing, based on the sensitivity and specificity for diagnosis of symptomatic hyperventilation in adults [12]. The questionnaire itself has neither been validated for use in asthma or for use in children. In the editorial accompanying the report by THOMAs et al. [2] which defined dysfunctional breathing in adults using Nijmegen questionnaire, KEELEY and OSMAN [13] have rightly pointed out that the sensitivity and specificity of the questionnaire in hyperventilation syndrome was shown in a study comparing a group of physician diagnosed non-asthmatic hyperventilators with a group of non-asthmatic normal controls.

DE GROOT et al. [11] specifically report the asthma control questionnaire was completed by parents for children younger than 12 years of age and by the children themselves if they were above 12 years of age, but they have not mentioned how the Nijmegen questionnaire was completed. Given that their study population was between 5 and 18 years of age, it would be important to know whether these questionnaires were administered by clinic staff or completed by parents or by children. Young children are likely to have difficulty comprehending some of the questions, e.g. confusion or loss of touch with reality, and parents may not be aware of symptoms in 
older children, e.g. tightness around mouth, blurred vision or feeling tense. Previous studies have shown poor correlation in parents' perception of their children's asthma control and the actual symptoms $[14,15]$.

There have also been suggestions that patients with dysfunctional breathing may have covert respiratory disease [16]. The correlation between poor asthma control and high Nijmegen scores (>23), as reported by DE GROOT et al. [11] could be real; however, there is considerable overlap between the symptoms suggestive of dysfunctional breathing and those clinicians associate with poor asthma control, such as: fast or deep breathing, shortness of breath, and difficulty breathing or taking a deep breath.

DE GROOT et al. [11] need to be congratulated for highlighting the possibility of dysfunctional breathing in children with asthma, especially in those with poorly controlled asthma. However, the results of the study should be taken with caution because of the possibility of several organic causes of wheeze in patients with poorly controlled asthma, like a missed foreign body [6]. In addition, the Nijmegen questionnaire requires validation for use in children with asthma.

There is no denying that clinicians see patients with functional overlay to their symptoms and obtaining a true estimate of the prevalence of conditions such as dysfunctional breathing would be valuable. The diagnosis of dysfunctional breathing in poorly controlled asthmatic children requires consideration and exclusion of other organic causes of wheezing.

\section{STATEMENT OF INTEREST}

None declared.

\section{REFERENCES}

1 Masoli M, Fabian D, Holt S, et al. The global burden of asthma: executive summary of the GINA dissemination committee report. Allergy 2004; 59: 469-478.
2 Thomas M, McKinley RK, Freeman E, et al. Prevalence of dysfunctional breathing in patients treated for asthma in primary care: cross sectional survey. BMJ 2001; 322: 1098-1100.

3 Thomas M, McKinley RK, Freeman E, et al. Breathing retraining for dysfunctional breathing in asthma: a randomised controlled trial. Thorax 2003; 58: 110-115.

4 Hagman C, Janson C, Emtner M. Breathing retraining - a five-year follow-up of patients with dysfunctional breathing. Respir Med 2011; 105: 1153-1159.

5 Niggemann B, Paul K, Keitzer R, et al. Vocal cord dysfunction in three children - misdiagnosis of bronchial asthma? Pediatr Allergy Immunol 1998; 9: 97-100.

6 Weinberger M, Abu-Hasan M. Pseudo-asthma: when cough, wheezing, and dyspnea are not asthma. Pediatrics 2007; 120: 855-864.

7 Niggemann B. How to diagnose psychogenic and functional breathing disorders in children and adolescents. Pediatr Allergy Immunol 2010; 21: 895-899.

8 de Groot EP. Breathing abnormalities in children with breathlessness. Paediatr Respir Rev 2011; 12: 83-87.

9 Grüber C, Lehmann C, Weiss C, et al. Somatoform respiratory disorders in children and adolescents-proposals for a practical approach to definition and classification. Pediatr Pulmonol 2012; 47: 199-205.

$10 \mathrm{Lu} \mathrm{Y,} \mathrm{Mak} \mathrm{KK,} \mathrm{van} \mathrm{Bever} \mathrm{HP,} \mathrm{et} \mathrm{al.} \mathrm{Prevalence} \mathrm{of} \mathrm{anxiety} \mathrm{and}$ depressive symptoms in adolescents with asthma: a meta-analysis and meta-regression. Pediatr Allergy Immunol 2012; 23: 707-715.

11 de Groot EP, Duiverman EJ, Brand PLP. Dysfunctional breathing in children with asthma: a rare but relevant comorbidity. Eur Respir J 2013; 41: 1068-1073.

12 van Dixhoorn J, Duivenvoorden HJ. Efficacy of Nijmegen Questionnaire in recognition of the hyperventilation syndrome. J Psychosom Res 1985; 29: 199-206.

13 Keeley D, Osman L. Dysfunctional breathing and asthma. BMJ 2001; 322: 1075-1076.

14 Dell SD, Foty R, Becker A, et al. Parent-reported symptoms may not be adequate to define asthma control in children. Pediatr Pulmonol 2007; 42: 1117-1124.

15 Kuehni CE, Frey U. Age-related differences in perceived asthma control in childhood: guidelines and reality. Eur Respir J 2002; 20: 880-889.

16 Morgan MD. Dysfunctional breathing in asthma: is it common, identifiable and correctable? Thorax 2002; 57: Suppl. 2, II31-II35. 